Liebe Kolleginnen und Kollegen,

wir freuen uns sehr, die erste modulare Fortbildung der AG im neuen Format und gleichzeitig die erste digitale Veranstaltung wissenschaftlich auszurichten zu dürfen. Die Weiterbildung der Radiologen und -innen im Thema Kinderradiologie liegt uns sehr am Herzen und die digitale Form erlaubt es, besonders viele anzusprechen. Wir haben für Sie ein breitgefächertes Programm zusammengestellt, das von Basiswissen bis hin zu hochkomplexen Themen reicht und auch das aktuellste Thema derzeit - virale Infektionen - mitumfasst. Natürlich hätten wir Sie gerne „live“ in Köln willkommen geheißen. Dies wird leider nicht möglich sein, aber wir sind uns sicher, dass wir Ihnen auch digital das besondere Flair unserer Domstadt vermitteln können!

Ihre

PD Dr. Friederike Körber, Dr. Martin Stenzel Wissenschaftliche Leitung

\title{
58. Jahrestagung (Kongress, Kurs, MTRA-Tagung) der Gesellschaft für Pädiatrische Radiologie, 23.-25.09.2021, Graz, Österreich
}
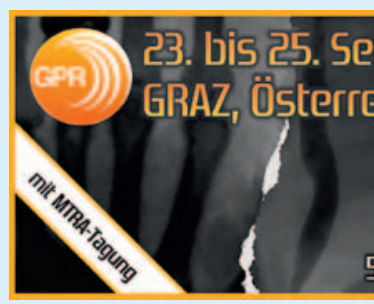

\section{Ein herzliches Grüß Gott zur GPR-Jahrestagung 2021!}

Die Gesellschaft für Pädiatrische Radiologie hat die Klinische Abteilung für Kinderradiologie der Medizinischen Universität Graz damit betraut, nach 1985 und 2010, nun auch 2021 die 58. Jahrestagung mit Fortbildungskurs auszurichten. Wir arbeiten schon mit großer Freude und Eifer daran.

Wir dürfen Sie herzlich zu diesem spannenden Kongress einladen, der unter dem Motto steht „Kinderradiologie Reloaded - im Zentrum oder Randerscheinung“. Das Programm ist so abgestimmt, dass ein Ausgleich zwischen diesen beiden Polen gefunden wird.

Um diesem Motto gerecht zu werden, werden wir die zentralen Themen wie Sonografie, Atemwegserkrankungen in allen Lebensabschnitten oder beispielsweise auch Uroradiologie aufgreifen. Andererseits werden aber auch Themen wie die Fragen des Neurochirurgen an die Kinderradiologie oder Bildgebung bei Retinoblastom behandelt werden.

Andere Themen die uns im Alltag immer wieder begegnen wie die EU-Datenschutzverordnung und deren Hintergründe sollen auch beleuchtet werden. Neue Bildgebende Methoden werden präsentiert werden.

Artificial Intelligence ist in aller Munde und wir wollen auch dieses Thema aufgreifen, jedoch in einer anderen Art und Weise als dies bisher geschehen ist. Eine Live-Demonstration eines lernenden Systems soll weitere Einblicke geben als auch wie man die Lernfähigkeit einer solchen Applikation beurteilt.

Selbstverständlich wird es genügend Zeit für Diskussionen und wissenschaftliche Beitrage geben, zu denen ich auch recht herzlich einlade. Bei entsprechender Anmeldung können wir auch eine Kinderbetreuung organisieren, sodass auch junge Eltern die Möglichkeit haben, einen entspannten Kongress zu genießen.

Der Fortbildungskurs wird als „roten Faden“ die Patientensicherheit haben - von Kontrastmitteln, „Good Praxis“ der Durchleuchtung als auch im MRT. Ein weiteres Highlight sind auswählbare Workshops am Nachmittag zu Themen wie Lungenultraschall, HerzMRT, angeborene Herzfehler im ThoraxRöntgen oder auch „How to publish a paper successfully“.

Der Gesellschaftsabend wird am 24.09.2021 in den Prunkräumlichkeiten der Jesuiten-Universität im Stadtzentrum stattfinden. Und wer die Kinderradiologie Graz kennt, weiß wie wir zu feiern wissen ich kann schon jetzt versprechen, dass niemand auf den Sesseln sitzen bleiben wird.

Die AG Kinderradiologie wird zusätzlich junge Kolleg*Innen mit aktiven Beiträgen mit Kostenbeiträgen unterstützen.

Wir dürfen Sie schon jetzt herzlich zu diesem Kongress einladen. Graz liegt im Herzen Europas und ist verkehrstechnisch an alle Möglichkeiten angebunden, wie Flugverbindungen, Bahnverbindungen, Autobahnen und auch durch den Flixbus.

Wir verbleiben mit der Hoffnung, Sie recht zahlreich in Graz begrüßen zu dürfen und werden alles daran setzen, den Kongress „Face2Face“ abhalten zu können.

Ihr Team der Klinischen Abteilung für Kinderradiologie der Medizinischen Universität Graz

Alle Informationen finden Sie auf der offiziellen Kongress-Homepage: www.gprjahrestagung.de

\section{ABSTRACTEINREICHUNG GEÖFFNET!}

Reichen Sie jetzt Ihr Abstract ein! Bis zum 1. Mai 2021 können Sie Ihr Abstract für die 58. Jahrestagung der GPR einreichen. Alle Informationen finden Sie auf www.gpr-jahrestagung.de 


\section{Elterninformation: Mein Kind muss ins MRT}

Ihr Kind soll zur Abklärung eine MRT-Untersuchung (auch „Kernspintomografie“ oder „Magnetresonanztomografie“ genannt) erhalten? Sie haben Fragen zu dieser Untersuchungstechnik? Sie wollen wissen, wie Sie sich und Ihr Kind am besten darauf vorbereiten können? Der neue Ratgeber der Gesellschaft für Pädiatrische Radiologie (GPR) gibt Ihnen Auskunft.

\section{Inhalt}

- Warum ist die MRT gut für die Untersuchung von Kindern geeignet?

- Wie funktioniert die MRT?

- Wie lange dauert eine Untersuchung?
- Bitte nicht bewegen! Achtung magnetisch!

- Darf ich während der Untersuchung meines Kindes im Raum bleiben?

- Was kann der/die Kinderradiolog*in auf den Bildern erkennen?

- Wie sollte man sich auf eine MRT-Untersuchung vorbereiten?

- Wie geht es nach der MRT-Untersuchung weiter?

Der Ratgeber wurde erstellt mit freundlicher Unterstützung der Deutschen Gesellschaft für Kinder- und Jugendmedizin e. V. (DGKJ) und dem Förderverein kind\&radiologie e. V.

Alle weiteren Informationen finden Sie auf kinder-radiologie.org > Fachinfos > Elterninfos sowie auf der Homepage der DGKJ > Eltern > DGKJ Elterninformationen > Mein Kind soll ins MRT.
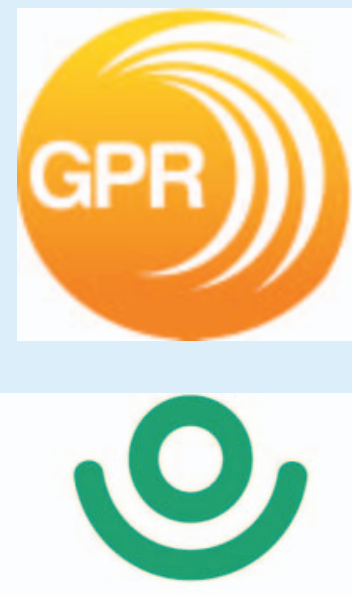

DEUTSCHE GESELLSCHAFT FÜR KINDER- UND JUGENDMEDIZIN e.V.

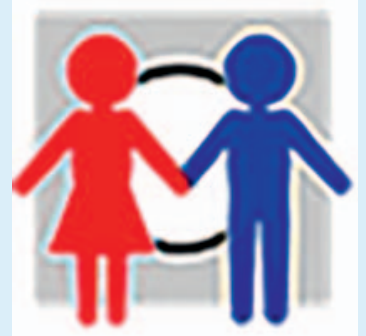

